A novel weighted method for layered SAW filters using slanted finger interdigital transducers

This article has been downloaded from IOPscience. Please scroll down to see the full text article.

2006 J. Phys. D: Appl. Phys. 39466

(http://iopscience.iop.org/0022-3727/39/3/007)

The Table of Contents and more related content is available

Download details:

IP Address: 140.112.113.225

The article was downloaded on 23/12/2008 at 02:20

Please note that terms and conditions apply. 


\title{
A novel weighted method for layered SAW filters using slanted finger interdigital transducers
}

\author{
Chih-Ming Lin, Yung-Yu Chen and Tsung-Tsong Wu \\ Institute of Applied Mechanics, National Taiwan University, Taipei, Taiwan, \\ Republic of China
}

Received 28 September 2005, in final form 13 December 2005

Published 20 January 2006

Online at stacks.iop.org/JPhysD/39/466

\begin{abstract}
In this paper, we propose a novel weighted method to improve the inclined pass-band problem that is intrinsic to surface acoustic wave (SAW) filters with slanted finger interdigital transducers (SFITs). By the dispersive characteristics of SAW in layered piezoelectric substrates, the present method could make the inclined pass-band flatter or the bandwidth wider. We derived a coupling-of-modes model for the calculation of the frequency responses of layered SAW devices with SFITs and calculated the dispersive characteristics of an AlN/silicon layered piezoelectric substrate, such as the phase velocity and the electromechanical coupling coefficient. Then we utilized the dispersive electromechanical coupling coefficient to flatten the inclined pass-band and the dispersive phase velocity to widen the bandwidth. Two designed examples of wide-band layered SAW filters using SFITs on AIN/silicon substrates are presented to prove the validity of the proposed method: one is designed to flatten the inclination of the pass-band; the other, to widen the bandwidth. Results show that the dispersions of layered piezoelectric media are favourable for improving the performance of SFIT-based SAW filters.
\end{abstract}

\section{Introduction}

Since telecommunication systems have recently reached a requirement for higher bit rates and more transmitted data, this has led to the urgent need for surface acoustic wave (SAW) filters with wider signal bandwidth and higher frequency, such as the apodized SAW filters or the SAW filters with slanted finger interdigital transducers (SFITs). The apodized SAW filter is not suitable for this application because of its poor sidelobe rejection in the stop-band and large ripples in the pass-band. By contrast, the SFIT-based SAW filter has a flat pass-band, an excellent linear phase across the pass-band and a small chip size; hence this type of SAW filter has been under extensive development in recent years.

The SFIT-based SAW filter was reported first by Hyodo et al [1] in Japan. Later, increasing research activities were concentrated on this kind of SAW filter in telecommunication applications due to its flat pass-band and excellent linear phase across the pass-band. Nevertheless, the SFIT-based SAW filter has some intrinsic problems that need to be solved, such as the pass-band inclination. In the 1980s, Campbell et al [2-5] proposed several methods to flatten the inclined pass-band, such as using shunt inductance, designing apodized slanted finger transducers and drawing curved fingers. In the 1990s, Yatsuda et al [6-10] demonstrated many useful techniques to realize SFIT-based SAW filters with wide-band and large side lobe suppression, low loss and low ripple, using the withdrawal-weighting method, the aperture weighting method and the floating electrodes slanted finger unidirectional transducer (FE-SFUDT) method. In addition, Balashov and Baek [11] employed the coupling-of-modes (COM) model to design matching circuits for SFIT-based SAW filters, and Martin and Steiner [12,13] proposed SAW filters with one-focus SFIT to save computation time on analysing conventional SFIT. It is worth noting that the wide-band feature of the SFIT SAW device has also led to several important applications, such as the nondestructive evaluation of thin films [14], actuating micro droplet [15] and measurement of band gaps in phononic crystals [16].

However, the authors of the above literature modified the geometric shapes of SFITs to improve the performances of 


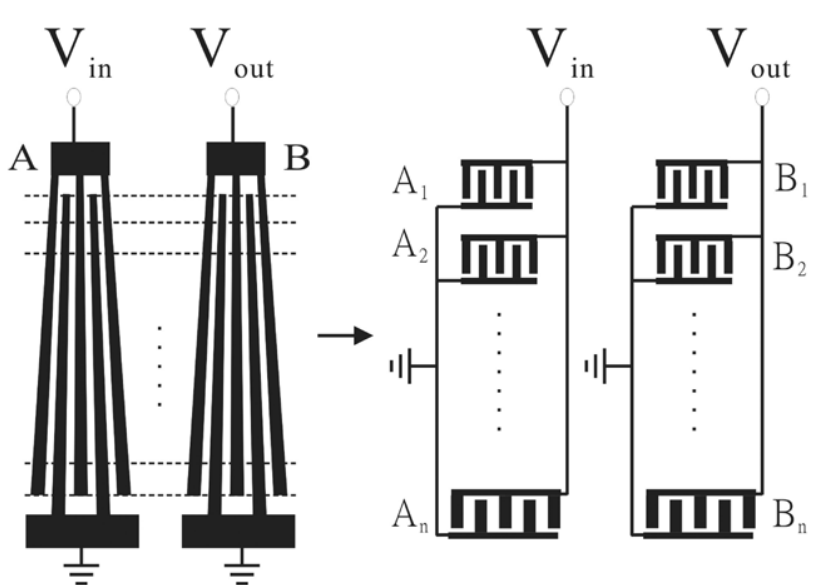

Figure 1. Schematics of a conventional SFIT SAW filter and its supposition of numerous sub-filters connected in parallel.

SFIT-based SAW filters, especially to flatten the inclined passband caused by the transducers' impedance. In this paper, we propose a novel design technique to solve the pass-band inclination problem by using a layered piezoelectric medium as the substrate. Primarily, we derived the COM model, valid for layered SAW devices with SFITs, to calculate the frequency responses of SFIT-based SAW filters based on the layered piezoelectric substrates; then we designed two wideband layered SAW filters with SFITs on AIN/silicon substrates. One is designed to verify the validity of the proposed method for flattening the inclined pass-band; the other is designed to widen the bandwidth.

\section{COM model for SAW filters with SFITs}

The COM model has been derived and utilized for analysing SAW filters with UNIFORM transducers on half-space and layered piezoelectric media $[17,18]$. When SAW filters are based on layered piezoelectric media, some COM parameters become frequency dependent because of the dispersive phase velocity and the dispersive electromechanical coupling coefficient; accordingly, these parameters have to be modified to fit the dispersive characteristics. In this section, we extend further the dispersive COM model proposed in [18] to establish a model for calculating the frequency responses of layered SFIT-based SAW devices.

Figure 1 shows the geometric illustration of a pair of SFITs. The periods of SFITs vary linearly along the axis perpendicular to the wave propagation direction. The shorterperiod section of a SFIT can excite higher frequency SAW and the longer-period section can excite lower frequency SAW; consequently, the SFIT-based SAW filter has a wideband frequency response. While we calculate the frequency response, the SFIT-based SAW filter is usually divided into $n$ sub-filters as shown in figure 1. If $n$ is large enough (generally, $35 \leqslant n \leqslant 100$ ), the tilt of the sub-filters can be neglected; that is, each sub-filter could be viewed as a filter with uniform transducers [2].

Accordingly we adopted the dispersive COM model to derive the relations between the bus-bar currents and the busbar voltages of sub-filters. For the $i$ th sub-filter, the bus-bar currents are related to the bus-bar voltages by the $\boldsymbol{Y}$-matrix and the relation can be expressed as $[11,19]$

$$
\left[\begin{array}{l}
I_{\mathrm{A}}^{i} \\
I_{\mathrm{B}}^{i}
\end{array}\right]=\left[\begin{array}{ll}
Y_{11}^{i} & Y_{12}^{i} \\
Y_{21}^{i} & Y_{22}^{i}
\end{array}\right]\left[\begin{array}{c}
V_{\mathrm{A}}^{i} \\
V_{\mathrm{B}}^{i}
\end{array}\right],
$$

where $I_{\mathrm{A}}^{i}$ and $I_{\mathrm{B}}^{i}$ are the bus-bar currents of the $i$-th input and output sub-filter, respectively. $V_{\mathrm{A}}^{i}$ and $V_{\mathrm{B}}^{i}$ are the busbar voltages of the $i$-th input and output sub-filter respectively. The elements of the $\boldsymbol{Y}$-matrix are expressed as

$$
\begin{gathered}
Y_{11}^{i}=P_{33}^{\mathrm{A}_{i}}+P_{11}^{\mathrm{B}_{i}}\left[\frac{P_{32}^{\mathrm{A}_{i}} P_{23}^{\mathrm{A}_{i}}}{1-P_{11}^{\mathrm{B}_{i}} P_{22}^{\mathrm{A}_{i}} \mathrm{e}^{-i 2 k L_{\mathrm{D}}^{i}}}\right] \mathrm{e}^{-j 2 k L_{\mathrm{D}}^{i},} \\
Y_{12}^{i}=\frac{P_{32}^{\mathrm{A}_{i}} P_{13}^{\mathrm{B}_{i}}}{1-P_{11}^{\mathrm{B}_{i}} P_{22}^{\mathrm{A}_{i}} \mathrm{e}^{-j 2 k L_{\mathrm{D}}^{i}}} \mathrm{e}^{-j k L_{\mathrm{D}}^{i}}, \\
Y_{21}^{i}=\frac{P_{23}^{\mathrm{A}_{i}} P_{31}^{\mathrm{B}_{i}}}{1-P_{11}^{\mathrm{B}_{i}} P_{22}^{\mathrm{A}_{i}} \mathrm{e}^{-j 2 k L_{\mathrm{D}}^{i}}} \mathrm{e}^{-j k L_{\mathrm{D}}^{i}}, \\
Y_{22}^{i}=P_{33}^{\mathrm{B}_{i}}+P_{22}^{\mathrm{A}_{i}}\left[\frac{P_{13}^{\mathrm{B}_{i}} P_{31}^{\mathrm{B}_{i}}}{1-P_{11}^{\mathrm{B}_{i}} P_{22}^{\mathrm{A}_{i}} \mathrm{e}^{-j 2 k L_{\mathrm{D}}^{i}}}\right] \mathrm{e}^{-j 2 k L_{\mathrm{D}}^{i}},
\end{gathered}
$$

where $k$ is the wave number of the SAW; $P_{x y}^{\mathrm{A}_{i}}$ and $P_{x y}^{\mathrm{B}_{i}}$ are the elements of the $\boldsymbol{P}$-matrix of the $\boldsymbol{i}$-th input and output sub-filter, respectively.

As mentioned above, a SFIT-based SAW filter is divided into $n$ sub-filters and treated as the connection of all sub-filters in parallel as shown in figure 1. Namely, the bus-bar voltage of SFIT is equal to the bus-bar voltage of each sub-filter and the bus-bar current of SFIT is equal to the summation of the busbar currents of all sub-filters; therefore, the bus-bar currents of input SFIT and output SFIT, $I_{\mathrm{A}}$ and $I_{\mathrm{B}}$, are expressed as

$$
I_{\mathrm{A}}=I_{\mathrm{A}}^{1}+I_{\mathrm{A}}^{2}+\cdots+I_{\mathrm{A}}^{n}=\sum_{i=1}^{n} I_{\mathrm{A}}^{i}
$$

and

$$
I_{\mathrm{B}}=I_{\mathrm{B}}^{1}+I_{\mathrm{B}}^{2}+\cdots+I_{\mathrm{B}}^{n}=\sum_{i=1}^{n} I_{\mathrm{B}}^{i},
$$

where $I_{\mathrm{A}}^{i}$ and $I_{\mathrm{B}}^{i}$ are the bus-bar currents of the $i$-th input and output sub-filter, respectively. Furthermore, the $\boldsymbol{Y}$-matrix of a SFIT-based SAW filter on a layered piezoelectric medium can be derived as

$$
\left[\begin{array}{c}
I_{\mathrm{A}} \\
I_{\mathrm{B}}
\end{array}\right]=\left[\begin{array}{ll}
Y_{11} & Y_{12} \\
Y_{21} & Y_{22}
\end{array}\right]\left[\begin{array}{l}
V_{\mathrm{A}} \\
V_{\mathrm{B}}
\end{array}\right],
$$

where $V_{\mathrm{A}}$ and $V_{\mathrm{B}}$ are the voltages of input SFIT and output SFIT, respectively. The elements of the $\boldsymbol{Y}$-matrix are expressed as

$$
Y_{x y}=\sum_{i=1}^{n} Y_{x y}^{i}, \quad x, y=1,2 .
$$

A SFIT-based SAW filter with different input/output pairs, as design- 1 in table 1 , is considered to demonstrate the pass-band inclination problem; each parameter in table 1 is illustrated in figure 2. As shown in figure 3, the measured frequency response is plotted with a solid line and the 
Table 1. SFIT SAW filters with three designs.

\begin{tabular}{|c|c|c|c|}
\hline Design no. & Design-1 & Design-2 & Design-3 \\
\hline Substrate & $\mathrm{YZ} \mathrm{LiNbO}_{3}$ & AlN/silicon & AlN/silicon \\
\hline $\begin{array}{l}\text { Thickness of } \\
\text { piezoelectric material }\end{array}$ & $\infty$ & $1 \mu \mathrm{m}$ & $3 \mu \mathrm{m}$ \\
\hline$\lambda_{\min }$ & $47.2 \mu \mathrm{m}$ & $20 \mu \mathrm{m}$ & $8.4 \mu \mathrm{m}$ \\
\hline$\lambda_{\max }$ & $69.6 \mu \mathrm{m}$ & $34 \mu \mathrm{m}$ & $10.4 \mu \mathrm{m}$ \\
\hline Input pairs & 20 pairs & 40 pairs & 40 pairs \\
\hline Output pairs & 30 pairs & 50 pairs & 50 pairs \\
\hline Metal thickness & $1500 \AA$ & $500 \AA$ & $500 \AA$ \\
\hline Propagation length & $5585.4 \mu \mathrm{m}$ & $2728.5 \mu \mathrm{m}$ & $834.6 \mu \mathrm{m}$ \\
\hline Aperture & $3000 \mu \mathrm{m}$ & $5000 \mu \mathrm{m}$ & $1000 \mu \mathrm{m}$ \\
\hline Maximum tilt angle & $6.44^{\circ}$ & $4.04^{\circ}$ & $2.89^{\circ}$ \\
\hline
\end{tabular}

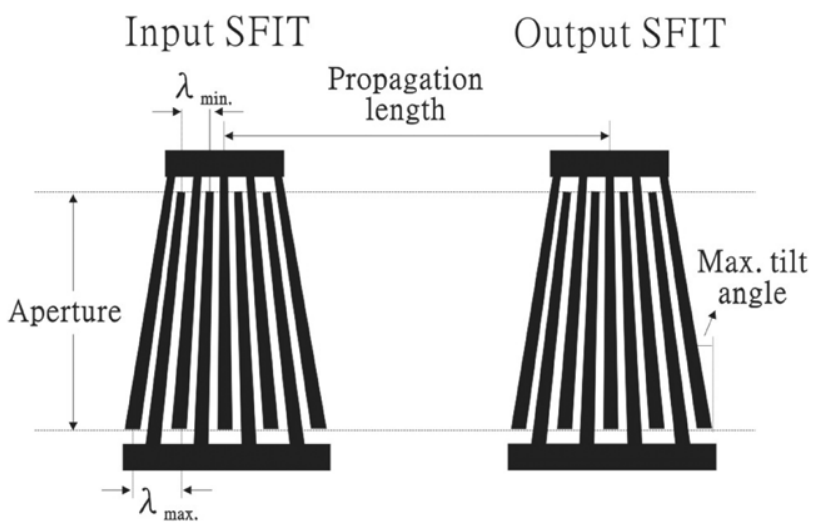

Figure 2. Illustration of designed parameters of a SFIT SAW filter.

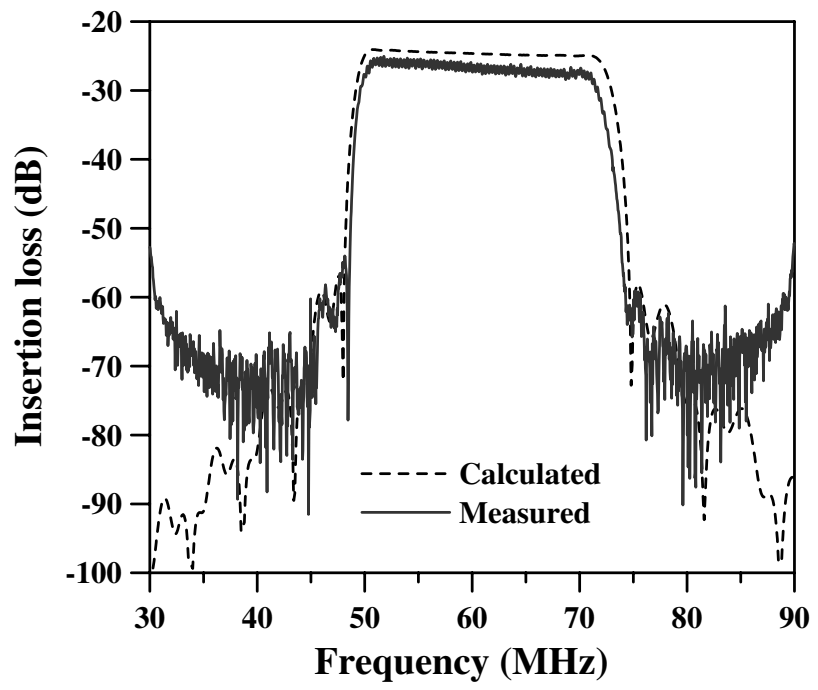

Figure 3. Calculated and measured frequency responses of the SFIT SAW filter of design-1 in table 1 .

calculated one is plotted with a dotted line. The measurement in this study was performed by the on-wafer measurement technology. From this figure, we observe that the calculated and measured frequency responses are in agreement except for the slight differences of insertion loss and ripples in the passband. The ripples are induced by finger reflection and triple transit echo (TTE); the slight mismatch of insertion loss may be caused by the misestimated propagation loss. It is worth noting that both the calculated and measured results have the obviously inclined pass-band. In the following sections, we

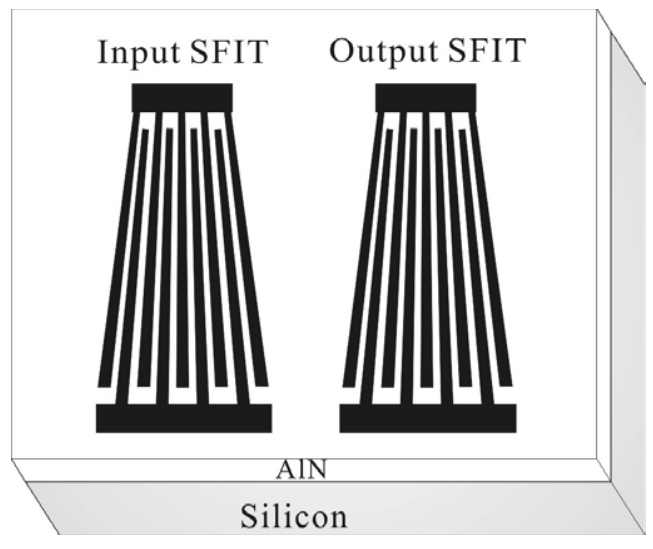

Figure 4. A novel SAW filter using SFITs on AlN/silicon.

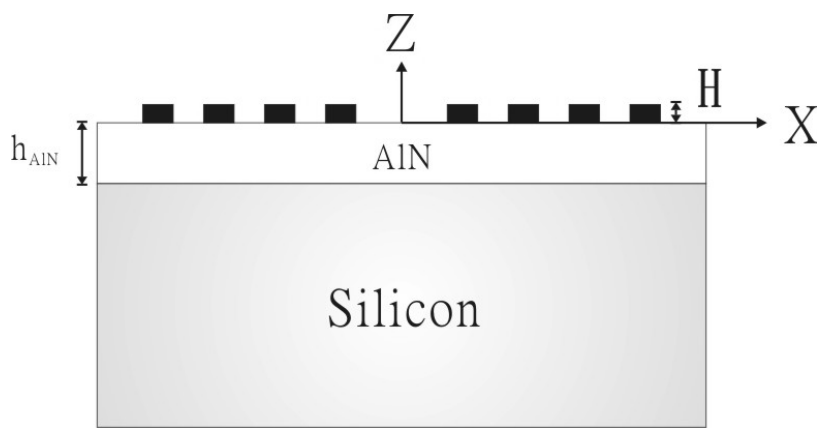

Figure 5. The perspective view of the SAW filter using SFITs on AlN/silicon.

will flatten it with the dispersive electromechanical coupling coefficient of the SAW in a layered piezoelectric medium.

\section{SAW propagation in AIN/silicon layered media}

In this paper, we propose a novel weighted method to compensate the pass-band inclination with the dispersive electromechanical coupling coefficient in a layered piezoelectric medium. To verify the validity of this method, we consider a SAW filter with SFITs on an AlN/silicon layered medium. Its configuration is shown in figure 4 and the side view is shown in figure 5 .

First, the dispersion characteristics of an AlN/silicon layered medium are studied based on the formulation in [18]. The epitaxial relationships are that the $(11 \overline{2} 0)$ plane of AIN is parallel to the surface of the (001) silicon substrate, and the SAW propagates in the plane normal to the $c$-axis of the AlN film and the (100) direction of the silicon substrate. All the related constants utilized in the calculations are from [20]. The calculation results are shown in figure 6: the solid line and the dotted line represent the dispersive phase velocity and the dispersive electromechanical coupling coefficient, respectively. In addition, the abscissa is the multiplication of frequency $f$ and thickness of AlN layer $h_{\mathrm{AlN}}$, the left ordinate is the phase velocity and the right ordinate is the electromechanical coupling coefficient. In contrast to SAW in a half-space substrate, the phase velocity and the electromechanical coupling coefficient of SAW in the layered substrate are no longer constant; rather, they are frequency dependent. 


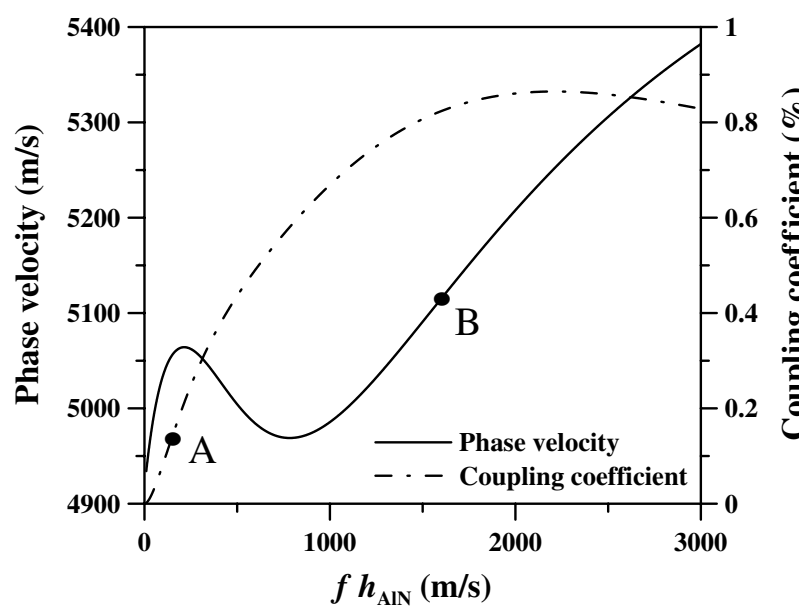

Figure 6. Calculated electromechanical coupling coefficient and phase velocity dispersion of a SFIT/AIN/silicon layered system.

Similarly to the phase velocity and the electromechanical coupling coefficient in a layered piezoelectric structure, some COM parameters-velocity change, reflection coefficient, transduction coefficient and static capacitance-become dispersive when we analyse layered SAW filters. In other words, as long as the dispersions of the COM parameters are considered, the COM model can be extended to analyse metal-grating strips and transducers deposited on a layered medium [18]. Furthermore, the thin film resistance [21] is assumed to be independent of frequency and the propagation loss of IDT/AIN/silicon is adopted from the experimental values in [22] in our study.

\section{Frequency responses of SFIT/AIN/silicon layered SAW filters}

In general, the larger the electromechanical coupling coefficient of a substrate, the smaller the insertion loss of a SAW filter based on it. In figure 6, we can find that the electromechanical coupling coefficient of AlN/silicon increases with $f h_{\text {AlN }}$ in the nearby region of point A; therefore, AlN/silicon layered piezoelectric media are very suitable for adoption to compensate the inclined pass-band.

A SFIT-based SAW filter of design- 2 in table 1 is considered to verify the validity of flattening the inclined pass-band with the dispersive electromechanical coupling coefficient. Figure 7 shows its calculated frequency response which is based on the COM model proposed in section 2: the dotted line represents the frequency response under nondispersion and the solid line is the frequency response under dispersion. When the dispersive effect is not considered, the pass-band slope of the frequency response is about $6 \mathrm{~dB} / 100 \mathrm{MHz}$; when it is considered, the slope becomes around $0.5 \mathrm{~dB} / 100 \mathrm{MHz}$. The results show that the inclined pass-band is successfully flattened by appropriately choosing the film thickness or SAW wavelength according to the dispersive electromechanical coupling coefficient. This proves the ability of this weighted method-the electromechanical coupling coefficient weighted method-to fatten the inclined pass-band with the dispersive electromechanical coupling coefficient of SAW in a layered piezoelectric medium.

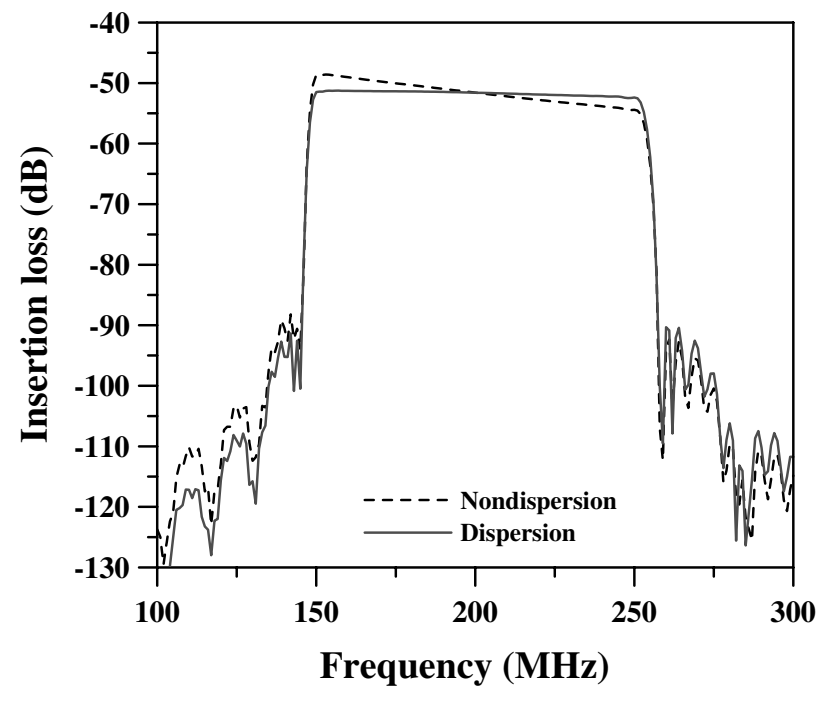

Figure 7. Calculated frequency response of a layered SAW filter using SFITs, designed as design-2 in table 1, based on non-dispersion and dispersion.

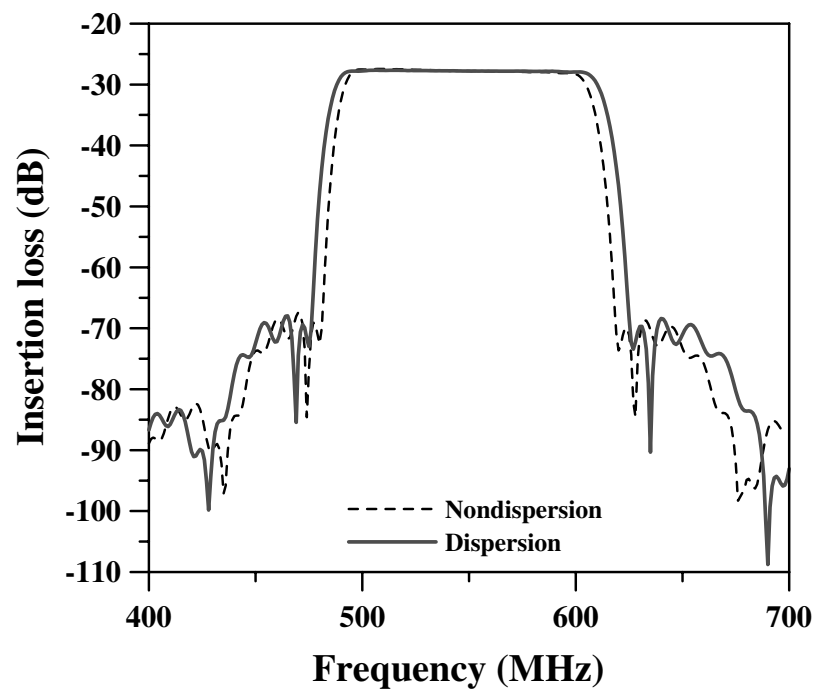

Figure 8. Calculated frequency response of a layered SAW filter using SFITs, designed as design-3 in table 1 , based on non-dispersion and dispersion.

In addition, the bandwidth and the thickness of AlN must be contemplated; otherwise the pass-band still inclines. For instance, if the thickness of AlN is thinner than $1 \mu \mathrm{m}$, the pass-band would still incline from the low frequency to the high frequency due to the less dispersive electromechanical coupling coefficient within the pass-band. On the contrary, if the thickness is larger than $1 \mu \mathrm{m}$, the pass-band would likewise incline but from the high frequency to the low frequency in this case due to a more dispersive electromechanical coupling coefficient within the pass-band.

The other layered SFIT SAW filter on AlN/silicon is designed, as shown in design-3 of table 1, to widen the bandwidth and its calculated frequency response is shown in figure 8 . The dotted line represents the frequency response under non-dispersion; the solid line, under dispersion. When the dispersive effect is not considered, the frequency response 
has a bandwidth of about $110 \mathrm{MHz}$; on the contrary, if it is concerned, the bandwidth is enlarged to $117 \mathrm{MHz}$. The increase of $7 \mathrm{MHz}$ in bandwidth is mainly attributable to the increase of phase velocity with $f h_{\mathrm{AlN}}$ in the nearby region of point $\mathrm{B}$ in figure 6 . This phenomenon is different from that of the SAW filters on $\mathrm{ZnO} /$ sapphire [18] since the phase velocity of SAW in $\mathrm{ZnO} /$ sapphire decreases with the product of the frequency and the $\mathrm{ZnO}$ thickness. Therefore, as long as we choose appropriately the film thickness and frequency to make the phase velocity increase with the product of frequency and film thickness, the bandwidth of a SFIT-based SAW filter can be widened with the dispersive phase velocity of a layered piezoelectric medium.

\section{Conclusion}

In this paper, we proposed a novel design technique to flatten the inclined pass-band of a SFIT-based SAW filter or to widen its bandwidth by using a layered piezoelectric medium as the substrate. We established a COM model for analysing SFIT-based SAW filters on layered piezoelectric media and then calculated the dispersive characteristics of SAW in an AlN/silicon layered medium, including the electromechanical coupling coefficient and the phase velocity. Finally, we also calculated the frequency responses of two SFIT-based SAW filters on AlN/silicon: one is designed to flatten the inclined pass-band with the dispersive electromechanical coupling coefficient and the other is designed to widen the bandwidth with the dispersive phase velocity. Results prove the validity of our proposed method; in other words, we can adopt the layered piezoelectric media as substrates to flatten the passband inclination of a SFIT SAW filter as well as to widen its bandwidth.

\section{Acknowledgments}

The authors would like to thank Dr C K Campbell for giving valuable suggestions. Financial support for this research from the National Science Council of Taiwan through NSC93-2218E-002-052 is gratefully acknowledged.

\section{References}

[1] Hyodo T, Yamanouchi K and Shibayama K 1968 The wide band excitation of elastic surface wave using the interdigital electrodes with variable pitches Proc. Acoust. Soc. Japan. Autumn Ann. Mtg. (Japan, 3-1-14) pp 193-4

[2] Campbell C K, Ye Y and Sferrazza PaPa J J 1982 Wide-band linear phase SAW filter design using slanted transducer fingers IEEE Trans. Son. Ultrason. SU-29 224-8

[3] Naraine J J and Campbell C K 1983 Wide band linear phase SAW filter using apodized slanted finger transducers Proc. IEEE Ultrasonics Symp. (Atlanta, GA) pp 113-16
[4] Slater N J and Campbell C K 1984 Improved modelling of wide-band linear phase SAW filters using transducers with curved fingers IEEE Trans. Son. Ultrason. SU-31 46-50

[5] Saw C B and Campbell C K 1987 Improved design of single-phase unidirectional transducers for low-loss SAW filters Proc. IEEE Ultrasonics Symp. (Denver, CO) pp 169-72

[6] Yatsuda H, Takeuchi Y and Yoshikawa S 1990 New design techniques for SAW filters using slanted-finger IDTs Proc. IEEE Ultrasonics Symp. (Honolulu, HI) pp 61-6

[7] Yatsuda H 1997 Design techniques for SAW filters using slanted finger interdigital transducers IEEE Trans. Ultrason. Ferroelect. Freq. Contr. 44 453-9

[8] Yatsuda H 1998 Design techniques for nonlinear phase SAW filters using slanted finger interdigital transducers IEEE Trans. Ultrason. Ferroelect. Freq. Contr. 45 41-7

[9] Yatsuda H 2000 Automatic computer-aided design of SAW filters using slanted finger interdigital transducers IEEE Trans. Ultrason. Ferroelect. Freq. Contr. 47 140-47

[10] Yatsuda H, Noguchi K and Yamanouchi K 2000 Surface acoustic wave filter using floating electrode slanted finger unidirectional transducers Japan. J. Appl. Phys. 39 3041-4

[11] Balashov S M and Baek K H 2000 Optimal design of wide band low loss SAW filters, using slanted interdigital transducers Proc. IEEE Ultrasonics Symp. (San Juan, PR) vol 1, pp 83-6

[12] Martin G and Steiner B 2001 SAW filters including one-focus slanted finger interdigital transducers Proc. IEEE Ultrasonics Symp. (Atlanta, GA) vol 1, pp 45-8

[13] Martin G and Steiner B 2003 SAW filters including one-focus slanted finger interdigital transducers IEEE Trans. Ultrason. Ferroelect. Freq. Contr. 50 94-8

[14] Wu T-T, Chen Y-Y, Huang G-T and Chang P-Z 2005 Evaluation of elastic properties of submicrometer thin films using slanted finger interdigital transducers J. Appl. Phys. 97073510

[15] Wu T-T and I-H Chang 2005 Actuating and detecting of micro droplet using slanted finger interdigital transducers $J$. Appl. Phys. 98024903

[16] Wu T-T, Wu L-C and Huang Z-G 2005 Frequency band-gap measurement of two-dimensional air/silicon phononic crystals using layered slanted finger interdigital transducers J. Appl. Phys. 97094916

[17] Abbott B P 1989 A coupling-of-modes model for SAW transducers with arbitrary reflectivity weighting $P h D$ Dissertation Department of Electrical Engineering at the University of Central Florida, Orlando FL

[18] Chen Y-Y, Wu T-T and Chou T-T 2004 Analysis of the frequency response of a dispersive IDT/ZnO/sapphire SAW filter using effective permittivity and the coupling of modes model J. Phys. D: Appl. Phys. 37 120-7

[19] Wu T-T and Lin C-M 2004 Analysis of SFIT SAW filters using coupling of modes model J. Chin. Inst. Eng. 27 973-9

[20] Auld B A 1990 Acoustic Fields and Waves in Solids (Florida: Krieger) pp 365-90

[21] Morgan D P 1985 Surface-Wave Devices for Signal Processing (New York: Elsevier) p 167

[22] Liaw H M and Hickernell F S 1994 SAW characteristics of sputtered aluminum nitride on silicon and gallium arsenide Proc. IEEE Ultrasonics Symp. (Cannes, France) pp 375-9 